\title{
TNF-Inhibitoren könnten vor schwerem Verlauf schützen
}

Die Mehrzahl der Rheumamedikamente ist auch bei einer Infektion mit SARS-CoV-2 sicher einsetzbar. Einige Wirkstoffe scheinen sogar vor schweren Verläufen der COVID-19-Erkrankung zu schützen. Einzelne Medikamente gegen entzündlich-rheumatische Erkrankungen stehen jedoch im Zusammenhang mit komplizierten Verläufen.

Medikamente gegen entzündlich-rheumatische Erkrankungen beeinflussen das Immunsystem. Einige erhöhen die Infektanfälligkeit. Dies kann auch den Verlauf einer COVID-19-Erkrankung beeinflussen. Deshalb untersuchten PD Dr. Anne Regierer und Dr. Martin Schäfer vom Programmbereich Epidemiologie am DRFZ und Dr. Rebecca Hasseli, Koordinatorin des COVID19-Rheuma Registers der DGRh, die Daten von 2274 Patient*innen mit einer entzündlichrheumatischen Erkrankung und einer SARS-CoV-2-Infektion. Das Register ist ein gemeinsames Projekt der DGRh und der Universität Gießen, dort geleitet von Prof. Dr. Ulf Müller-Ladner. Die Kommission COVID-19-Register der DGRh wertet die Registerdaten kontinuierlich aus. Auch die Studie von Regierer [1] ist im Rahmen dieser Kommissionstätigkeit entstanden.

Die Forschenden des DRFZ und der DGRh kategorisierten schwere Verläufe in drei Gruppen: ambulant behandelt (1), stationär aufgenommen (2) und invasiv beatmet oder verstorben (3). „Bei der großen Mehrheit verlief die Infektion glücklicherweise unkompliziert", berichtet Dr. Hasseli. Dies waren $78 \%$. Leider verstarben 83 Patient*innen an COVID-19, dies sind 3,6\% der zu diesem Zeitpunkt im Register Erfassten.

„Für die Rheumapatient*innen zeigte sich“, so Regierer, „dass wie bei nicht von Rheuma Betroffenen schwere Verläufe oft mit höherem Alter, männlichem Geschlecht und zusätzlich bestehenden Herz-Kreislauf-Erkrankungen verbunden sind." Erheblichen Einfluss nehme aber auch die Aktivität der rheumatischen Erkrankung: Patient* innen mit einer mittleren bis hohen Krankheitsaktivität hatten ein deutlich höheres Risiko für einen schwereren COVID-19-Verlauf als solche mit "stabiler" Erkrankung. Bei Patient*innen, die eine hohe Krankheitsaktivität hatten und zusätzlich auch Glukokortikoide einnahmen, war das Risiko für einen komplizierten Infektionsverlauf noch höher.

In der Gruppe der Immunsuppressiva, die zur Behandlung rheumatischer Erkrankungen eingesetzt werden, gingen einzelne etwas häufiger mit einem schwereren COVID-19-Verlauf einher, andere wiederum nicht. Die Analyse der einzelnen Substanzen ergab aus Sicht der Expert*innen interessante Unterschiede: während das Biologikum Rituximab mit einem schwereren Verlauf von COVID-19 assoziiert war und die so genannten JAK-Inhibitoren dieses Risiko moderat zu erhöhen scheinen, waren die häufig eingesetzten TNF-Inhibitoren dagegen eher mit einem milderen Verlauf der SARS-CoV-2-Infektion assoziiert. „Wir müssen diese Daten umsichtig interpretieren, da es sich um eine Querschnittserhebung handelt, die keine unmittelbaren Schlüsse im Sinne von wenn-dann zulässt", betont Prof. Dr. Christof Specker, Vorstandsmitglied der DGRh und Sprecher der Kommission. So kommen etwa JAK-Inhibitoren und auch Rituximab häufiger bei komplizierteren Rheuma-Verläufen zum Einsatz.

\section{Das wichtigste ist die Kontrolle der Krankheitsaktivität}

Als Fazit aus dieser Arbeit zieht Regierer, dass die von vielen Menschen mit Rheuma eingenommenen TNF-Inhibitoren bei einer COVID-Infektion sicher sind. Sie könnten eventuell sogar vor schweren Verläufen schützen. Das wichtigste bleibe die Kontrolle der Krankheitsaktivität.

Deshalb sollten Betroffene auch auf keinen Fall Medikamente selbständig absetzen. Die behandelnden Rheumatolog*innen müssen die Therapie sorgfältig abwägen. Hierzu zählt auch die konsequente Aufklärung über die Impfung und eine möglichst hohe Impfrate unter Rheumapatient*innen.

\section{Literatur \\ 1. Regierer AC, Hasseli R, Schäfer M, et al TNFi is associated with positive outcome, but JAKi and rituximab are associated with negative outcome of SARS-CoV-2 infection in patients with RMD. https://rmdopen.bmj.com/ content/7/3/e001896.full}

Hinweis des Verlags. Der Verlag bleibt in Hinblick auf geografische Zuordnungen und Gebietsbezeichnungen in veröffentlichten Karten und Institutsadressen neutral.

\section{rheuma plus $2022 \cdot 21: 31$}

https://doi.org/10.1007/s12688-

022-00497-4

(c) The Author(s), under exclusive licence to Springer-Verlag GmbH Austria, ein Teil von Springer Nature 2022
Quelle: Presseaussendung der Deutschen Gesellschaft für Rheumatologie e.V. (DGRh) 